\title{
A multistudy examination of organizational stressors, emotional labor, burnout, and turnover in sport organizations
}

R. J. Larner, C. R. D. Wagstaff, R. C. Thelwell, and J. Corbett 
Abstract

While a growing body of research has examined the types of organizational stressors

3 encountered by individuals and their allied responses, little is known about how such

4 individuals manage their emotional responses to these stressors or the consequences of such

5 behaviors. This paper presents novel findings from two studies examining the moderating role

6 that emotional labor plays in the relationship between the frequency of organizational stressor

7 experience, burnout, turnover intentions, and actual turnover in sport. In study 1, participants (n

$8=487$ ) completed measures of organizational stressors (OSI-SP), emotional labor (ELS),

9 burnout (ABQ), and turnover intentions. In study 2, a 6-month longitudinal design was used to

10 examine measures of organizational stressors (OSI-SP), emotional labor (ELS), turnover

11 intentions, and actual turnover. Study 1 showed that surface acting moderated the relationship

12 between the frequency of organizational stressors and burnout in sport. Further, surface acting

13 acted as an important mechanism through which burnout mediated the relationship between the

14 frequency of organizational stressors and turnover intentions. Study 2 showed that surface

15 acting moderated the relationship between the organizational stressor frequency and turnover

16 intentions - but not actual turnover - over time. These results highlight the importance of surface

17 acting in understanding how individuals respond to organizational stressors encountered in

18 sport, expanding our understanding of the positive and negative responses component of the

19 meta-model of stress, emotions and performance. These findings also highlight potentially

20 deleterious emotion-management behaviors that practitioners might consider when aiming to

21 support individuals encountering organizational stressors in sport.

23 Keywords: longitudinal, stress, surface acting, well-being, dropout, emotion regulation 
A Multi-Study Examination of Organizational Stressors, Emotional Labor, Burnout, and Turnover in Sport Organizations

In line with the growing acknowledgement of the importance of organizational issues in elite sport (see, e.g., Fletcher \& Wagstaff, 2009; Wagstaff \& Larner, 2015), a healthy body of work has emerged to indicate that participation in competitive sport is typically characterized by a wide range of demands that could lead to a disruption in performance and impaired health and well-being (see also Fletcher et al., 2006). Moreover, a category of demands that are particularly prevalent and problematic for athletes are those associated with the organization within which they operate (see for reviews, Arnold \& Fletcher, 2012b; Fletcher \& Wagstaff, 2009; Fletcher et al., 2012). Indeed, sport organizations have become increasingly complex, comprising of various intra-group, inter-group, and organizational levels, with all members of the organization contributing to its functioning and effectiveness (Fletcher \& Wagstaff, 2009). The present research aims to answer Fletcher and Wagstaff's (2009) call for research incorporating a range of stakeholders (e.g., athletes, coaches, managers, sport science and medicine staff) in psychological research in sport organizations.

With regards to the prevalence of these demands, sport performers have been found to experience and recall more organizational stressors than those associated with competitive performances (Hanton et al., 2005). Indeed, the area of organizational stress and well-being has received more research attention than any other area within organizational psychology in sport (Wagstaff \& Larner, 2015). Much of this research has been conceptually aligned with the metamodel of stress and emotions (Fletcher \& Fletcher, 2005), which posits stress as a process that exists between an individual and their environment. In line with this model, Fletcher et al. (2006) defined organizational stress in sport as, "an ongoing transaction between an individual and the environmental demands associated primarily and directly with the organization within which he or she is operating" (p. 329). To elaborate, recent research indicates that individuals respond to a range of environmental demands (i.e., organizational stressors) in a variety of 
1 emotional, behavioral, and attitudinal ways (Fletcher et al., 2012), that may have diverse

2 consequences for burnout (Tabei et al., 2012), dissatisfaction (Noblet et al., 2003), negative

3 emotions (Fletcher et al., 2012), impaired preparation for and performance in major

4 competitions (Gould et al., 1999) and substantially affected health and well-being (DiBartolo \&

5 Schaffer, 2002).

In keeping with Fletcher et al.'s (2006) definition of organizational stress, much

7 research has sought to identify the types of organizational stressors encountered by individuals

8 in sport (e.g., Arnold et al., 2014; Fletcher et al., 2010; Fletcher et al., 2012; Kristiansen et al.,

9 2012). In a synthesis of this research, Arnold and Fletcher (2012a) developed a taxonomic

10 classification of stressors in sport that included four main categories (viz. leadership and

11 personnel, cultural and team, logistical and environmental, and performance and personal

12 issues). In line with this taxonomy, Arnold et al. (2013) presented a series of studies describing

13 its development and validation of the Organizational Stressor Indicator for Sport Performers

14 (OSI-SP). More recently, Arnold et al. (2014) used the OSI-SP to identify demographic

15 differences (i.e., gender, competition level, and team or individual sport type) in performers'

16 experiences of organizational stressors, providing a stimulus for future research to examine

17 additional moderating variables in the stress process. In addition to the identification and

18 measurement of organizational stressors in sport, researchers have explored individuals'

19 responses to these demands including, but not limited to, athlete burnout (e.g., Fletcher et al.,

20 2012; Kristiansen et al., 2011; Tabei et al., 2012).

21 Within sport, athlete burnout has been described within a psychosocial framework

22 comprising three key components: physical and emotional exhaustion (i.e., perceived depletion

23 of energy due to the demands of sport participation and performance), sport devaluation (i.e.,

24 diminished interest in and negative attitude towards sports participation), and reduced athletic

25 accomplishment (i.e., unfulfilled goals and a sense of constantly falling short of performance

26 standards) (Raedeke, 1997). While a number of conceptual approaches have been put forth to 
1 explain burnout (see, for review, Cresswell \& Eklund, 2006a), recently an integrated model of

2 burnout (see Gustafsson et al., 2011) has been proposed that incorporates antecedents, early

3 signs, key dimensions, consequences, personality factors, coping and the environment. Indeed,

4 identifying antecedents that contribute to burnout in sport is of central importance to current

5 models, with researchers (see Gustafsson et al., 2008) pointing to numerous situational and

6 organizational antecedents of burnout in elite athletes.

The importance of investigating burnout within sport organizations stems from the

8 detrimental impact it can have on health, well-being, and performance. Indeed, burnout has

9 been associated with negative affective, cognitive, motivational, and behavioral consequences

10 such as decreased performance, overtraining, reduced sense of accomplishment, depressed

11 mood, feelings of helplessness, diminished motivation and eventual withdrawal from sport

12 (Cresswell \& Eklund, 2006b; Goodger et al., 2007; Gustafsson et al., 2008, 2011).

13 Although it would appear that stressors are an important component of the burnout

14 process, not all individuals who experience stress will burnout or withdraw from sport

15 (Raedeke, 1997). Therefore, to better understand why individuals report different outcomes to

16 similar organizational stressors, research might examine the role of potential moderating and

17 mediating variables, including personal and situational characteristics and various cognitive,

18 emotional, and attitudinal phenomena (see Arnold et al., 2014: Didymus \& Fletcher, 2012,

19 2014; Fletcher et al., 2006; Hanton et al., 2012; Sarkar \& Fletcher, 2014; Tabei et al., 2012).

20 Indeed, a growing body of research (e.g., Fletcher et al., 2006, 2012b; Fletcher \& Wagstaff,

21 2009; Lane et al., 2012; Tamminen \& Crocker, 2013; Wagstaff et al., 2012a, 2012b) has

22 highlighted the importance of emotion regulation (i.e., monitoring, evaluating, and modifying

23 emotional reactions) for performance and well-being outcomes in sport organizations. For

24 example, Wagstaff et al. (2012a) noted the importance of regulating one's emotions and

25 aligning them with the expectations and social norms of the organization. 
The need to manage emotions to fit with the expectations and social norms of the sport organization (see Wagstaff et al., 2012a, 2012b) aligns with the concept of emotional labor

3 (Hochschild, 1983). Emotional labor has been defined as, "the process of regulating both

4 feelings and expressions for the organizational goals" (Grandey, 2000). To elaborate,

5 individuals will display organizationally-desirable emotional expressions regardless of the

6 emotions that they are actually experiencing at that time (Grandey, 2000; Hochschild, 1983).

7 According to Hochschild (1983), there are two main ways to engage in emotional labor:

8 through surface acting (i.e., only regulating emotional expressions) or deep acting (i.e.,

9 consciously modifying feelings to express the desired emotions). Research conducted in non-

10 sport organizations has shown deep acting to be associated with more positive outcomes

11 compared to surface acting (see, for review, Hülsheger \& Schewe, 2011). To elaborate, deep

12 acting, which occurs earlier in the emotion-generation process and involves reappraisal, has

13 been shown to have positive outcomes such as greater personal accomplishment, job

14 satisfaction, personal efficacy, and performance for employees (Brotheridge \& Grandey, 2002;

15 Grandey, 2003). Conversely, surface acting, which occurs later in the emotion-generation

16 process, has been associated with more negative outcomes such as turnover intentions, actual

17 turnover, reduced job performance, and depersonalisation (Brotheridge \& Lee, 2003; Chau et

18 al., 2009; Goodwin et al., 2011). Further, emerging research (e.g., Lee \& Chelladurai, 2015;

19 Rogers et al., 2014) indicates that the management of emotions through surface acting may lead

20 to negative well-being outcomes such as depressive symptoms and burnout.

21 In line with the potentially deleterious implications of emotion management, a body of

22 research exists, which collectively indicates that withdrawal behaviors may be an outcome of

23 emotional labor (e.g., Brotheridge \& Lee, 2003; Grandey, 2000). Moreover, Chau et al. (2009)

24 argued that surface acting may contribute to increased turnover of employees due to its effortful

25 nature, creation of emotional dissonance, and associated emotional exhaustion. Within sport,

26 Wagstaff (2014) found emotional suppression (i.e., surface acting) resulted in a 3.3\% 
1 performance decrement on a 10k cycling time trial. In light of the potential ramifications and

2 the requirements on individuals to display various emotions as part of their role and the

3 necessity to regulate their own and others' emotions to maintain interpersonal relationships and

4 improve organizational functioning (see Wagstaff et al., 2012a, 2012b), it is surprising that no

5 research has examined the interaction of such phenomena.

The purpose of the studies presented here was to examine the relationship between

organizational stressors and burnout in sport, with particular focus on the potential moderating role of surface acting, and the perceived impact that these relationships have on subsequent

9 turnover intentions. The main study hypotheses were as follows: the frequency of

10 organizational stressors will be positively related to burnout in individuals operating in sport

11 organizations (H1); there will be a positive relationship between burnout and turnover

12 intentions in individuals operating in sport organizations (H2); the relationship between the

13 frequency of organizational stressors and burnout in sport will be moderated by surface acting

14 such that the relationship between the frequency of organizational stressors and burnout will be

15 stronger in individuals with high levels of surface acting (H3); and burnout will mediate the

16 relationship between the frequency of organizational stressors and turnover intentions. This

17 indirect effect will be moderated by surface acting such that the indirect relationship between

18 the frequency of organizational stressors and turnover intentions through burnout will be

19 positive and stronger in individuals with high levels of surface acting (H4). Figure 1 provides a

20 visual representation of the hypothesised research model.

[FIGURE 1 AROUND HERE]

\section{Study 1}

\section{Method}

\section{Participants and Procedure}

487 participants from a variety of individual and team sports (e.g., football, cricket, netball) took part in the study $(N=47)$. The age of participants ranged from 16 to 60 years $(M=$ 
$122.8, S D=8.45)$. At the time of data collection, all participants were operating within sport organizations as either athletes $(n=389)$, coaches $(n=74)$, performance directors $(n=7)$, or sport scientists and medics $(\mathrm{n}=17)$. Within their organizations, participants were operating at a variety of levels ranging from club $(n=183)$, county $(n=121)$, regional $(n=62)$, national $(n=$ 75), and international $(\mathrm{n}=46)$. Participants were recruited via opportunity sampling and online distribution. A link to a web-based online questionnaire or a paper hard-copy of the questionnaire was sent out to all participants. Prior to data collection, a favourable ethical opinion was received and information about the nature of the study and issues of confidentiality and anonymity were explained to all participants.

\section{Measures}

A range of validated questionnaires were used to address the research hypotheses and measured organizational stressors, emotional labor, burnout, and turnover intentions respectively. Both online and paper versions of the questionnaire were piloted prior to the main study but as this did not reveal any deficiencies in the design, format or length of the questionnaire, no changes were made.

Organizational Stressor Indicator for Sport Performers (OSI-SP). The 23-item OSISP (Arnold et al., 2013) was used to assess the frequency of a range of organizational stressors encountered by sport individuals. Arnold et al. (2013) stated using the frequency scale alone would be adequate for researchers requiring a shorter version of the indicator. Therefore, items were measured in relation to the frequency of each organizational stressor on a Likert scale ranging from 0 (never) to 5 (always). The five subscales on the OSI-SP were: goals and development, logistics and operations, team and culture, coaching, and selection. For the present study, all five frequency subscales showed acceptable internal consistency ( $\alpha=.66$ to $.84)$.

Emotional labor Scale (ELS). The 15-item self-report ELS (Brotheridge \& Lee, 2003) measures six facets of emotional display, including the frequency, intensity, and variety of the 
1 emotional display, and surface and deep acting. Higher scores on a 5-point Likert scale from 1

2 (never) to 5 (always) indicate greater emotional labor. Questionnaire items where adapted to the

3 context of study with the word "job" being replaced with the word "role"; for example, "on an

4 average day, how frequently do you express particular emotions needed for your role”. Internal

5 consistency Cronbach's alpha for the surface acting subscale was .76.

Athlete Burnout Questionnaire (ABQ). Athlete burnout was assessed using the 15-

7 item ABQ (Raedeke \& Smith, 2001). The ABQ comprises of three subscales designed to assess

8 reduced sense of accomplishment, sport devaluation, and emotional/physical exhaustion. Items

9 were measured on a 5-point Likert scale ranging from 1 (almost never) to 5 (almost always).

10 Higher total average scores on the ABQ indicated a greater degree of burnout. Internal

11 consistency Cronbach's alphas for the three subscales were .75 for reduced accomplishment,

12.78 for sport devaluation, and .88 for physical and emotional exhaustion.

13 Coach Burnout Questionnaire (CBQ). Coaches, performance directors, and sport

14 scientists and medics included in the sample completed the CBQ. The CBQ is a 15-item

15 measure that is reworded to assess burnout in coaches. The original ABQ question stems are

16 altered for the CBQ to reflect coaching rather than athletic participation in sport. For example,

17 "I'm accomplishing many worthwhile things in [sport]" is changed to "I'm accomplishing many

18 worthwhile things coaching [sport]." Examination of fit, clarity and the meaning of revised

19 items has found the CBQ to have appropriate content validity and modification of items, with

20 acceptable Cronbach's alphas (between .81 and .94) being reported (Harris \& Ostrow, 2008).

21 The CBQ was selected as it discriminates between dimensions of burnout in a sports context

22 that previous measures of burnout do not (Lundkvist et al., 2014). The CBQ was also deemed

23 appropriate to use for performance directors and sport scientists and medics given their

24 substantive coaching nature of their roles. The title of "coach" is commonly used

25 interchangeably for performance directors (e.g., head coach) and support staff (e.g., strength

26 and conditioning coach) (see Wagstaff, 2016). 
Turnover intentions. Turnover intentions were assessed using the three items (Kim \&

2 Stoner, 2008): "In the next few months I intend to leave this organization", "In the next few

3 years I intend to leave this organization", and "I occasionally think about leaving this

4 organization". Items are rated on a 7-point Likert scale ranging from 1 (strongly disagree) to 7

5 (strongly agree). Kim and Stoner (2008) reported a Cronbach's alpha for internal consistency

6 alpha of .76. For the present study, internal consistency was found to be acceptable with a

7 Cronbach's alpha of .77.

\section{Data analysis}

In order to investigate whether the effect of organizational stressors on burnout varied in

10 magnitude and nature as a function of surface acting, a simple moderation analysis was used. In

11 addition, to examine whether the effect of organizational stressors on turnover intentions

12 through burnout varied as a function of surface acting, a moderated mediation analysis was used

13 (Hayes, 2013). Traditional techniques to test for moderation and mediation suffer from several

14 problems including low statistical power and the inability to test multiple proposed moderators

15 or mediators together (Hayes, 2012). Therefore, the present study used Hayes's (2012)

16 PROCESS macro, with 1000 bootstrap resamples and $95 \%$ confidence intervals to test indirect

17 effects for significance at different values of the moderator (i.e., surface acting). This

18 regression-based path analytic framework allows the input of data, configuration and estimation

19 of two and three way interactions in moderation models.

\section{Results}

\section{Preliminary analyses}

Table 1 shows the means, standard deviations, and intercorrelations for all study

23 variable dimensions. There was a positive relationship found between the frequency of

24 organizational stressors and mean burnout $(r=.32, p<.001)$. Surface acting was also positively

25 related to burnout $(r=.31, p<.001)$ and turnover intentions item 3, "I occasionally think about 
1 leaving this organization" $(r=.14, p<.01)$. In addition, mean burnout was positively related to

2 turnover intentions $(r=.37, p<.001)$.

[TABLE 1 AROUND HERE]

\section{Testing for moderation}

The simple moderation results are presented in Table 2. Consistent with hypothesis 3 , surface acting moderated the relationship between the frequency of organizational stressors and burnout $\left(F(3,483)=33.03, p=<.001, R^{2}=.20\right)$. For every one unit increase in stressor frequency, there was a .95 increase in burnout $b=.95, t(483)=5.67, p<.001$ ) and for every

9 one unit increase in surface acting, there was a .87 increase in burnout $(b=.87, t(483)=5.77, p$

$10<.001)$. The interaction between stressor frequency and surface acting was $b=.46, t(483)=$ $11 \quad 3.02, p<.05$.

\section{[TABLE 2 AROUND HERE]}

To illustrate the nature of this interaction, the relationship between the frequency of

14 organizational stressors, surface acting, and burnout is displayed graphically in Figure 2 (with one standard deviation above and below the mean of surface acting). Interaction slopes for stressor frequency predicting burnout showed that at low levels of surface acting burnout scores increased by $.54(b=.54, t(483)=2.36, p<.01)$ compared to athletes who reported high levels of surface acting, for whom burnout scores increased by $1.37(b=1.37, t(483)=6.65, p<.001)$.

\section{Testing for moderated mediation}

A moderated-mediation model was used to test whether the indirect effect of the

frequency of organizational stressors on turnover intentions through burnout is moderated by

surface acting (Figure 3). There was a conditional indirect effect of the frequency of

organizational stressors on turnover intentions through mean burnout (index of moderatedmediation $=.27,95 \%$ CI $[.10, .47])$. Specifically, the indirect effect of organizational stressors on turnover intentions through burnout was positive and increased with higher levels of surface 
acting (Table 3). The conditional direct effect was also moderated, as indicated by a statistically significant interaction (see Hayes, 2012). Indeed, the relationship between the frequency of organizational stressors and mean burnout was positive and significant when surface acting was

4 high $(b=.70, p<.05)$ but non-significant when equal to its mean $(b=.42, p=.12)$ or when

5 surface acting was low $(b=.16, p=.66)$. Thus, the mediational effect of burnout in the

6 relationship between the frequency of organizational stressors and turnover intentions were

7 stronger in those with high levels of surface acting.

[FIGURE 3 AROUND HERE]

\section{[TABLE 3 AROUND HERE]}

\section{Study 1 Discussion}

This study provides the first empirical examination of emotional labor in the context

12 of organizational stressors and burnout in sport and offers a valuable insight into the role these

13 constructs might play in such organizations. These findings serve to highlight the importance of

14 surface acting in understanding how individuals respond to organizational stressors they

15 encounter in sport. Indeed, at comparable stressor levels, surface acting increases burnout and

16 turnover intention. One explanation for this may be that surface acting, which requires

17 expressive suppression of socially undesirable emotions is effortful and drains limited mental

18 resources (e.g., Richards \& Gross, 2000). These self-regulatory cognitive and emotional

19 resources are important for the intra- and inter-personal processes that demand emotion

20 regulation within sport organizations (Wagstaff et al., 2012a, 2012b; Wagstaff et al., 2013).

21 Hence, the level of self-regulatory resources (i.e., sufficient or depleted) may account for the

22 different well-being and performance outcomes (e.g., burnout and turnover intentions) for

23 individuals performing emotional labor. Therefore, practitioners might encourage the avoidance

24 of potentially deleterious surface acting when working with individuals in sport.

\section{Study 2}


Study 1 examined surface acting as a moderator in the relationship between

organizational stressors, burnout, and turnover intentions in sport, and as such, provides a first empirical examination of mechanistic factors (i.e., emotional labor) that influence the organizational stress-response process in sport and extends previous work by providing an insight into how such variables influence both psychosocial (i.e., burnout) and behavioral intentions (i.e., turnover) outcomes. Nevertheless, it was beyond the scope of study 1 to examine how organizational stressors impacted actual turnover in sport. Therefore, we reiterate Fletcher et al. (2006) and Arnold et al.'s (2013) call for a longitudinal approach to better capture the complex, ongoing nature of organizational stressors.

Voluntary turnover from sport organizations is a salient issue given the negative effect it can have on replacement recruitment and training, operational functioning, and morale of the remaining members. In elite sport the turnover of talent due to environmental demands will affect team climate, culture, stability, and functioning. At the non-elite level, turnover from sport organizations is likely to impact participation rates and, where chronic, will threaten the survival of amateur sport organizations. Further, as alluded to in Study 1, surface acting may contribute to turnover due to its effortful nature and association with burnout (Brotheridge \& Grandey, 2002; Grandey, 2003). Therefore, and in an attempt to extend the findings of Study 1, the purpose of Study 2 was to examine the influence of surface acting in the relationship between organizational stressors, turnover intentions, and actual turnover in sport. Hence, a longitudinal design was employed to examine whether surface acting moderated the relationship between organizational stressors from time 1 and turnover intentions and actual turnover at time 2 , which was six months after time 1 . To our knowledge, there have not been any longitudinal studies that have investigated the stress-turnover relationship in sport. We hypothesised that the relationship between organizational stressors at time 1 and a) turnover intentions at time 2 and b) actual turnover will be moderated by surface acting such that the 
1 relationship between organizational stressors and a) turnover intentions b) actual turnover will

2 be stronger in individuals reporting high levels of surface acting.

\section{Method}

\section{Participants and procedure}

In total, 90 participants from a range of individual and team sports (e.g., athletics,

6 football, hockey) were recruited for this study $(n=28)$. The participants age ranged from 17 to

760 years $(M=26.70, S D=10.98)$. All participants were operating within sport organizations as

8 athletes $(n=65)$, coaches $(n=16)$, performance directors $(n=4)$, and or sport scientists and

9 medics $(n=5)$. Within their organizations, participants were operating at a variety of levels

10 ranging from club $(n=26)$, county $(n=21)$, regional $(n=12)$, national $(n=17)$, and

11 international $(\mathrm{n}=14)$. Either an online or paper hard-copy of a questionnaire including the OSI-

12 SP, ELS, and turnover intentions was sent to all participants to be completed. After

13 approximately 6 months (+/- 2 weeks), the same questionnaire pack was sent out to all

14 participants again with one additional item to measure actual turnover. Prior to data collection, a

15 favourable ethical opinion was received and information about the nature of the study and

16 issues of confidentiality and anonymity were explained to all participants.

\section{Measures}

The measures employed in study 1 for org stress (OSI-SP), emotional labor (ELS) and

19 turnover intentions, were again used for study 2. In addition, actual turnover was measured

20 using a single-item question: "I am no longer part of this sport organization".

\section{Data analysis}

As with Study 1, a simple moderation analysis was used to ascertain whether the effect

23 of the frequency of organizational stressors from time 1 on turnover intentions and actual

24 turnover from time 2 varied in magnitude and nature as a function of surface acting. The present

25 study used Hayes's (2012) PROCESS macro, with 1000 bootstrap resamples and 95\% 
1 confidence intervals to test indirect effects for significance at different values of the moderator

2 (i.e., surface acting).

\section{Results}

4 Preliminary analysis

5

Table 4 shows the means, standard deviations, and intercorrelations for study variable dimensions. The mean values reported were in accordance with extant literature. For the main study variables, there was a positive relationship found between the frequency of organizational stressors (time 1$)$ and surface acting $(r=.24, p<.05)$ but not turnover intentions (time 2$)$ or actual turnover (time 2). Turnover intentions (time 1 and time 2) were positively related to actual turnover $(r=.27, p<.05 ; r=.46, p<.001)$.

\section{[TABLE 4 AROUND HERE]}

Dependent t-tests showed a significant increase in the scores between turnover intentions at time $1(M=6.74, S D=4.31)$ to turnover intentions at time $2(M=8.27, S D=$ $5.05) ; t(89)=-2.82, p<.01$. There was a small but non-significant decrease in reports of the frequency of organizational stressors from time $1(M=1.45, S D=.74)$ to time $2(M=1.36, S D$ $=.74) ; t(89)=.10, p>.05$.

\section{Testing for moderation}

The relationship between the frequency of organizational stressors (time 1) and turnover intentions (time 2$)$ was moderated by surface acting $\left(F(3,86)=3.65, p=<.01, R^{2}=.11\right)$ supporting Hypothesis 5a. Indeed, the interaction of stressor frequency and surface acting on turnover intentions was $b=2.41, t(86)=2.66, p<.01$. Interaction slopes for stressors predicting turnover intentions showed that at high levels of surface acting turnover intention scores increased by $2.11(b=2.11, t(86)=2.39, p<.01)$, and for performers reporting low levels of surface acting there was not a significant increase in turnover intention scores $(b=.-2.01, t(86)$ $=-1.54, p=.13)$. However, although 14 out of 90 participants had left their sport organization by time 2 , the moderation results for actual turnover showed an insignificant interaction effect 
$1 \quad(b=.54,95 \% \mathrm{CI}[-.62,1.48], Z=.81, p>.05)$. Hence, the relationship between the frequency

2 of organizational stressors (time 1) and actual turnover (time 2) was not moderated by surface

3 acting and the findings were not consistent with Hypothesis $5 b$ (see Figures 4 and 5).

\section{[FIGURE 4 AROUND HERE]}

\section{[FIRGURE 5 AROUND HERE]}

\section{Study 2 Discussion}

This study provides the first longitudinal examination into the influence of the

frequency of organizational stressors and emotional labor on behavioral outcomes in sport. The main finding was that surface acting positively moderated the relationship between the frequency of organizational stressors (time 1) and turnover intention (time 2) whereby, the relationship between the frequency of organizational stressors (time 1) and turnover intentions (time 2) was only significant at higher levels of surface acting. Nevertheless, surface acting did not moderate the relationship between the frequency of organizational stressors (time 1) and actual turnover. The findings indicate that at comparable levels of organizational stressors experienced, those reporting greater emotional labor express an increased desire to leave their sport organization after six months, yet do not necessarily turnover.

Although intentions have been found to be the best predictors of behavior (Armitage \& Conner, 2001), this relationship can vary considerably. Indeed, the theory of planned behavior (Ajzen, 2002) proposes the intention-behavior relationship may itself be moderated. That is, the relationship between turnover intentions and actual turnover might be stronger in certain individuals and circumstances than others (e.g., those with greater perceived behavioral control, self-efficacy). For example, performers remaining in their current organization despite expressing a desire to leave may not be able to due to a lack of available and appropriate alternatives. Indeed, performers may want to leave their organization but resolve not to do so due to financial repercussions, distance to alternative organizations, or fears regarding reemployment. There is scope for future studies to explore these possible explanations further 
1 and to differentiate between performers (e.g., demographic differences) for whom remaining in

2 their organization may be more of a significant concern.

From an organization-level perspective, turnover can have negative consequences for

4 replacement recruitment and training, participation rates, stability, and functioning of the

5 organization. However, for individual performers, turnover from their organization may be a

6 positive outcome. For example, a coach or young athlete may decide to leave their organization

7 in order to progress their career or skill level elsewhere (see for review, Wylleman, Alfermann,

8 \& Lavallee, 2004). Indeed, to gain a more complete understanding on turnover in sport, future

9 research in this area should explore deeper into the different positive and negative reasons

10 leading to turnover intentions and actual turnover among key stakeholders in sport, which could

11 also be viewed in terms of dropout, career transitions, and career termination.

12 Despite the observation that turnover intention was not found to be a strong predictor

13 of actual turnover, the finding that turnover intention increased over time indicates that

14 performers were psychologically disengaged from their sport organization. Further, this study

15 identified that organizational stressor frequency and surface acting are among the factors that

16 drive psychological disengagement (i.e., turnover intention) in sport and provides a rationale for

17 developing targeted organizational and emotion regulation interventions to prevent further

18 psychological withdrawal.

\section{General Discussion}

In an attempt to significantly advance the organizational stressor theory and research

21 in sport by moving beyond the identification, appraisal, and consequences of organizational stressors, the studies presented here examined emotional labor as a moderator in the stress

23 process using both cross-sectional and longitudinal designs.

Our results show that frequency of organizational stressors was positively associated with all three dimensions of burnout in sport. This finding is consistent with Tabei et al. (2012), who reported a positive relationship between organizational stressors and burnout in football 
1 players. Such findings also reinforce the notion that the organization of sport can create

2 climates associated with higher incidences of burnout for individuals (Cresswell \& Eklund,

3 2006b; Gould et al., 1996). Given the emerging findings pertaining to the important role

4 emotional phenomena play in sport organizations (Wagstaff et al., 2012a, 2012b, 2013;

5 Wagstaff, 2014), it is perhaps not surprising that organizational-stressor dimensions were most

6 strongly correlated to physical and emotional exhaustion burnout scores in the present research.

7 This observation also lends support to the assertion that physical and emotional exhaustion

8 captures the 'core meaning' of burnout (see Cropanzano et al., 2003).

The results of this study significantly advance previous research by highlighting the

10 role of emotional labor as a moderating variable in the relationship between organizational

11 stressor frequency and burnout, as well as offering a first insight into such dynamics in sport.

12 The finding that surface acting moderates turnover intentions through burnout and turnover

13 intentions is salient because such findings significantly extend extant cross-sectional findings in

14 this domain and provides empirical support for several hypotheses of the meta-model of stress

15 and emotions (Fletcher \& Fletcher, 2005). Nevertheless, it is noteworthy that the relationship

16 between organizational stressors, surface acting, and actual turnover was not significant. To

17 determine why some individuals remain in their organization despite showing signs of burnout

18 and stating turnover intentions, there is a need to examine sport-related attitudes, such as

19 commitment, identity and engagement. Indeed, Jackson et al. (2014) found organizational

20 commitment mediated attrition rates in adolescent groups in sport. In the same study, Jackson et

21 al. (2014) developed a sport-specific measure of organizational commitment providing an excellent opportunity for future research to understand engagement, and intentions to turnover

23 or actual turnover in sport organizations (Wagstaff \& Larner, 2015).

The findings reported here suggest that emotional labor has significant explanatory

25 potential for psychosocial dynamics and outcomes in sport, specifically, that surface acting is

26 likely to lead to negative outcomes such as burnout and turnover intentions. Hence, it would 
1 appear that the use of surface acting is maladaptive and should be discouraged, yet we are

2 reluctant to make such conclusions or recommendations at this time. To elaborate, in light of

3 the negative outcomes associated with surface acting observed in the present studies, one might

4 question why individuals employ such strategies. In attempting to fathom such behavior,

5 previous research on organizational stressors in sport (see Hanton et al., 2012) might offer

6 insight. Indeed, Hanton et al., (2012) found that when encountering organizational stressors in

7 sport, individuals perceived them to be largely negative, and appraised themselves to have little

8 control and limited resources to cope with such demands. Such findings offer insight into why

9 individuals might employ surface acting; that is, because they do not think they can control or

10 resolve the demand through problem-focused action, they supress their emotional response to it.

11 Therefore, we encourage researchers to examine the cognitive determinants of surface acting in

12 order to better understand why individuals use such strategies despite the associated negative

13 wellbeing consequences. In terms of our reticence to advise the universal avoidance of surface

14 acting, we would add that the consequences of acute verses chronic surface acting are not well

15 understood, and it is possible that there are times when surface acting might be an effective

16 short-term strategy (e.g., to avoid interpersonal conflict). Clearly, further research is required to

17 better understand the complexity of emotional labor in sport organizations.

18 In addition to examining the complexity of the emotional labor process in sport,

19 researchers might seek to develop and evaluate emotion-regulation interventions for promoting

20 well-being and retaining talent and participation numbers in sport organizations. For example,

21 in a non-sport sample, Parkinson and Totterdell (1999) trained employees to use either

22 engagement strategies (i.e., direct attention towards current mood and challenges) or social

23 support strategies (i.e., divert attention away from the current situation), with those employees

24 who used engagement strategies to experience more positive moods were better at withstanding

25 emotional demands than those taught social support strategies. Further, there exists a body of

26 research in non-sport organizations evaluating the effectiveness of burnout prevention 
1 interventions. In a review of this literature, Awa et al., (2010) observed that the majority of

2 interventions were directed at the individual level (68\%), with $8 \%$ being aimed at organizational

3 change, and $24 \%$ were a combination of both. The authors concluded their review by arguing

4 that a combination of both intervention types should be further investigated, optimized and

5 practiced.

We perceive several strengths aligned with the present research. First, the

7 examination of organizational stressors, well-being outcomes and turnover extends quantitative

8 research in organizational psychology in sport. Further, the use of a longitudinal design to

9 predict the relationships between these variables over time also provides a novel contribution to

10 this literature. Nevertheless, as with all studies, there are limitations of the present research. One

11 limitation of the present research was the absence of appraisal data. According to the meta-

12 model of stress, appraisals play a key role in the stress process. Nonetheless, as appraisals are

13 highly individualised, these phenomena are difficult to measure using existent quantitative

14 methods and empirical examinations of organizational stress appraisals remain a key area for

15 future research (cf. Didymus \& Fletcher, 2012; Hanton et al., 2012). The absence of multilevel

16 analyses allowing for handling of the potentially clustered nature of the data is also a potential

17 limitation. However, very few participants were from the same sport or organization as others,

18 and therefore, we do not believe that data were skewed by organizational-level variables. In

19 addition, the OSI-SP was developed and validated with a specific population in mind (i.e.,

20 athletes) and therefore, required slight modification when used with other populations (i.e.,

21 coaches, managers, sport scientists and medics). To aid future research wanting to identify and

22 measure organizational stressors among other key stakeholders operating within sport, there is a

23 need to develop and validate the OSI for other sport populations. Finally, it was beyond the

24 scope of the present research to assess intensity and duration of stressors in addition to

25 frequency using the OSI-SP. Therefore, another potential area for future research would be to 
1 investigate these dimensions, particularly in relation to the experience of burnout and turnover

2 intention.

3 Perspectives

In putting these findings into perspective, the present studies significantly advance

5 current theory and research and empirically link organizational stressors and burnout in sport.

6 Further, the present studies advance emotional theory and research by identifying surface acting

7 as a moderating variable in the relationships between the frequency of organizational stressors,

8 burnout, and turnover intentions. These findings have the potential to assist sport organizations

9 to change individuals' experiences of organizational stressors and emotional labor and

10 subsequently reduce individuals' burnout and their desire to leave their organization. 


\section{References}

2 Ajzen I. Perceived behavioral control, self-efficacy, locus of control, and the theory of planned behavior. J Appl Soc Psychol 2002: 32: 665-683.

4 Armitage CJ., Conner M.. Efficacy of the theory of planned behavior: a meta-analytic review. Br J Soc Psychol 2001: 40: 471-499.

6 Arnold R., Fletcher D. A research synthesis and taxonomic classification of the organizational 7 stressors encountered by sport performers. J Sport Exerc Psychol 2012a: 34: 397-429.

8 Arnold R., Fletcher D. Psychometric issues in organizational stressor research: a review and 9 implications for sport psychology. Meas Phys Edu Exerc Sci 2012b: 16: 81-100.

10 Arnold R., Fletcher D. Daniels, K. Development and validation of the Organizational Stressor 11 Indicator for Sport Performers (OSI-SP). J Sport Exerc Psychol 2013: 35: 180-196.

12 Arnold R., Fletcher D. Daniels, K. Demographic differences in sport performers' experiences of 13 organizational stressors. Scand J Med Sci Sports 2014: 26: 348-358.

14 Awa WL, Plaumann M., Walter U. Burnout prevention: a review of intervention programs. Patient Educ Couns 2010: 78: 184-190.

16 Brotheridge CM., Grandey AA. Emotional labor and burnout: comparing two perspectives of “people work.” J Vocl Behav 2002: 60: 17-39.

18 Brotheridge CM., Lee RT. Development and validation of the Emotional Labor Scale. J Occup Organ Psychol 2003: 76: 365-379.

20 Chau SL., Dahling, JJ., Levy, PE., Diefendorff, JM. A predictive study of emotional labor and 21 turnover. J Organ Behav 2009: 30: 1151-1163.

22 Cresswell SL., Eklund RC. Current and Future Directions in Athlete Burnout. In S Hanton SD 23 Mellalieu (Eds.), Literature reviews in sport psychology (pp. 91-126). New York: Nova Science 2006a.

25 Cresswell SL., Eklund RC. The nature of player burnout in rugby: key characteristics and attributions. J Appl Sport Psychol 2006b: 18: 219-239. 
1 Cropanzano R., Rupp DE., Byrne ZS. The relationship of emotional exhaustion to work attitudes, job performance, and organizational citizenship behaviors. J Appl Sport Psychol 2003: 88: $160-169$.

4 DiBartolo PM., Schaffer C. A comparison of female college athletes and nonathletes: eating discorder symptomatology and psychological well-being. Sport Psychol 2002: 24: 33-41.

6 Didymus FF., Fletcher D. Getting to the heart of the matter: a diary study of swimmers' appraisals of organisational stressors. J Sport Sci: 2012: 30: 1375-1385.

8 Didymus FF., Fletcher D. Swimmers' experiences of organizational stress: Exploring the role of $9 \quad$ cognitive appraisal and coping strategies. J Clin Sport Psychol 2014: 8: 159-183.

10 Fletcher D., Fletcher J. A meta-model of stress, emotions and performance: Conceptual

11 foundations, theoretical framework, and research directions. J Sport Sci 2005: 23: 15712158.

13 Fletcher D., Hanton S., Mellalieu SD. (2006) An organizational stress review: conceptual and 14 theoretical issues in competitive sport. In S. Hanton \& S. D. Mellalieu (Eds.), Literature 15 reviews in sport psychology (pp. 321-374). New York: Nova Science.

16 Fletcher D., Hanton S., Mellalieu S., Neil R. A conceptual framework of organizational stressors. Scand J Med Sci Sports 2010: 22: 545-557.

18 Fletcher D., Hanton S., Wagstaff CRD. Performers' responses to stressors encountered in sport organisations. J Sport Sci 2012: 30: 349-58.

20 Fletcher D., Wagstaff CRD. Organizational psychology in elite sport: Its emergence, application 21 and future. Psychol Sport Exerc 2009: 10(4): 427-34.

22 Goodger K., Gorely T., Lavallee D., Harwood C. Burnout in sport: a systematic review. Sport Psychol 2007: 21: 127-151.

24 Goodwin RE., GrothM., Frenkel SJ. Relationships between emotional labor, job performance, and turnover. J Voc Behav 2011: 79: 538-548. 
1 Gould D., Guinan D., Greenleaf C., Mudbery R., Peterson K. Factors affecting Olympic performance: perceptions of athletes and coaches from more and less successful teams. Sport Psychol 1999: 13: 371-394.

4 Gould D., Tuffey S., Udry E., Loehr J. Burnout in competitive junior tennis players. 11:

5 Qualitative content analysis and case studies. Sport Psychol 1996: 10: 341-366.

6 Grandey AA. Emotion regulation in the workplace: a new way to conceptualize emotional labor. J 7 Occup Health Psych 2000: 5: 95-110.

8 Grandey AA. When "the show must go on": Surface acting and deep acting as determinants of 9 emotinal exhaustion and peer-rated service delivery. Acad Manage J 2003: 46: 86-96.

10 Gustafsson H., Hassmén P., Kenttä G., Johansson M. A qualitative analysis of burnout in elite 11 Swedish athletes. Psychol Sport Exerc 2008: 9: 800-816.

12 Gustafsson H., Kenttä G., Hassmén P. Athlete burnout: an integrated model and future research 13 directions. Int Rev Sport Exerc Psych 2011: 4: 3-24.

14 Hanton S., Wagstaff CRD., Fletcher D. Cognitive appraisals of stressors encountered in sport organizations. Int J Sport Exerc Psych 2012: 10: 276-289.

16 Harris BS., Ostrow AC. Coach and athlete burnout: The role of coaches' decision-making style. In 17 SR Bakere (Ed.), Hot topics in sports and athletics (pp. 143-157). Hauppage, NY: Nova 18 Science Publishers, Inc 2008.

19 Hayes AF. PROCESS: A versatile computational tool for observed variable mediation, moderation, and conditional process modeling [White paper] 2012.

21 Hayes AF. Introduction to mediation, moderation, and conditional process analysis: A regression-based approach. New York: Guilford Press 2013.

23 Hochschild AR. The managed heart: commercialization of human feelings. Berkeley: Univeristy of California Press 1983.

25 Hülsheger UR., Schewe AF. On the costs and benefits of emotional labor: a meta-analysis of three decades of research. J Occup Health Psychol 2011: 16: 361-389. 
1 Jackson B., Gucciardi DF., Dimmock JA. Toward a multidimensional model of athletes' commitment to coach-athlete relationships and interdependent sport teams: a substantivemethodological synergy. J Sport Exerc Psychol 2014: 36: 52-68.

4 Kim H., Stoner M. Burnout and turnover intention among social workers: Effects of role stress, $5 \quad$ job autonomy and social support. Admin Soc Work 2008: 32: 5-25.

6 Kristiansen E., Murphy D., Roberts GC. Organizational stress and coping in U.S. professional 7 soccer. J Appl Sport Psychol 2012: 24: 207-223.

8 Kristiansen E., Roberts GC., Sisjord MK. Coping with negative media content: The experiences of 9 professional football goalkeepers. Int J Sport Exerc Psychol 2011: 9: 295-307.

10 Lane AM., Beedie CJ., Jones MV., Uphill MA., \& Devonport TJ. The BASES expert statement on 11 emotion regulation in sport. J Sport Sci 2012: 30: 1189-1195.

12 Lee YH., \& Chelladurai P. Affectivity, emotional labor, emotional exhaustion, and emotional 13 intelligence in coaching. J Appl Sport Psycho 2015: 32: 1-16.

14 Lundkvist E., Stenling A., Gustafsson H., Hassmén P. How to measure coach burnout: an 15 evaluation of three burnout measures. Meas Phys Edu Exerc Sci 2014: 18: 209-226.

16 Noblet A., Rodwell J., Mcwilliams J. Predictors of the strain experienced by professional Australian footballers. J Appl Sport Psychol 2003: 15: 184-193.

18 Parkinson B., Totterdell P. Classifying affect-regulation strategies. Cognition Emotion 1999: 13: 277-303.

20 Raedeke TD. Is athlete burnout more than just stress? A sport commitment perspective. J Sport Exerc Psychol 1997: 19: 396-417.

22 Raedeke TD., Smith AL. Development and preliminary validation of an athlete burnout measure. J Sport Exerc Psychol 2001: 23: 281-306.

24 Richards JM., Gross JJ. Emotion regulation and memory: the cognitive costs of keeping one's cool. J Pers Soc Psychol 2000: 79: 410-424. 
1 Rogers ME., Creed PA., Searle J. Emotional labor, training stress, burnout, and depressive symptoms in junior doctors. J Vocat Edu Training 2014: 66: 232-248.

3 Sarkar M., Fletcher D. Psychological resilience in sport performers: a review of stressors and protective factors. J Sport Sci 2014: 32: 1419-1434.

5 Tabei Y., Fletcher D., Goodger K. The relationship between organizational stressors and athlete burnout in soccer players. J Clin Sport Psychol 2012: 6: 146-165.

7 Tamminen K., Crocker P. I control my own emotions for the sake of the team": Emotional selfregulation and interpersonal emotion regulation among female high-performance curlers. Psychol Sport Exerc 2013: 14: 737-747.

10 Wagstaff CRD. (2016) Coaching through organizational change: the influence of leadership 11 succession events. In Thelwell RC, Harwood C and Greenlees I (Eds.), The psychology of 12 sports coaching: Research and practice (pp. 68-83): Routledge, Oxford.

13 Wagstaff CRD. Emotion regulation and sport performance. J Sport Exerc Psychol 2014: 36: 401412.

15 Wagstaff CRD., Fletcher D., Hanton S. Exploring emotion abilities and regulation strategies in sport organizations. Sport Exerc Perf Psychol 2012a: 1: 268-282.

17 Wagstaff CRD., Fletcher D., Hanton S. Positive organizational psychology in sport: an ethnography of organizational functioning in a national sport organization. J Appl Sport Psychol 2012b: 24: 26-47.

20 Wagstaff CRD., Hanton S., Fletcher D. Developing emotion abilities and regulation strategies in a sport organization: An action research intervention. Psych Sport Exerc 2013: 14: 476-487.

22 Wagstaff CRD., Larner RJ. Organizational psychology in sport: Recent developments and a research agenda. In SD Mellalieu., S Hanton (Eds.), Contemporary reviews in sport psychology (pp. 91-11). Abingdon, UK: Routledge 2015.

25 Wylleman P., Alfermann D., Lavallee D. Career transitions in sport: European perspectives. Psychol Sport Exerc 2004: 5: 7-20. 
8

10

11

12

13

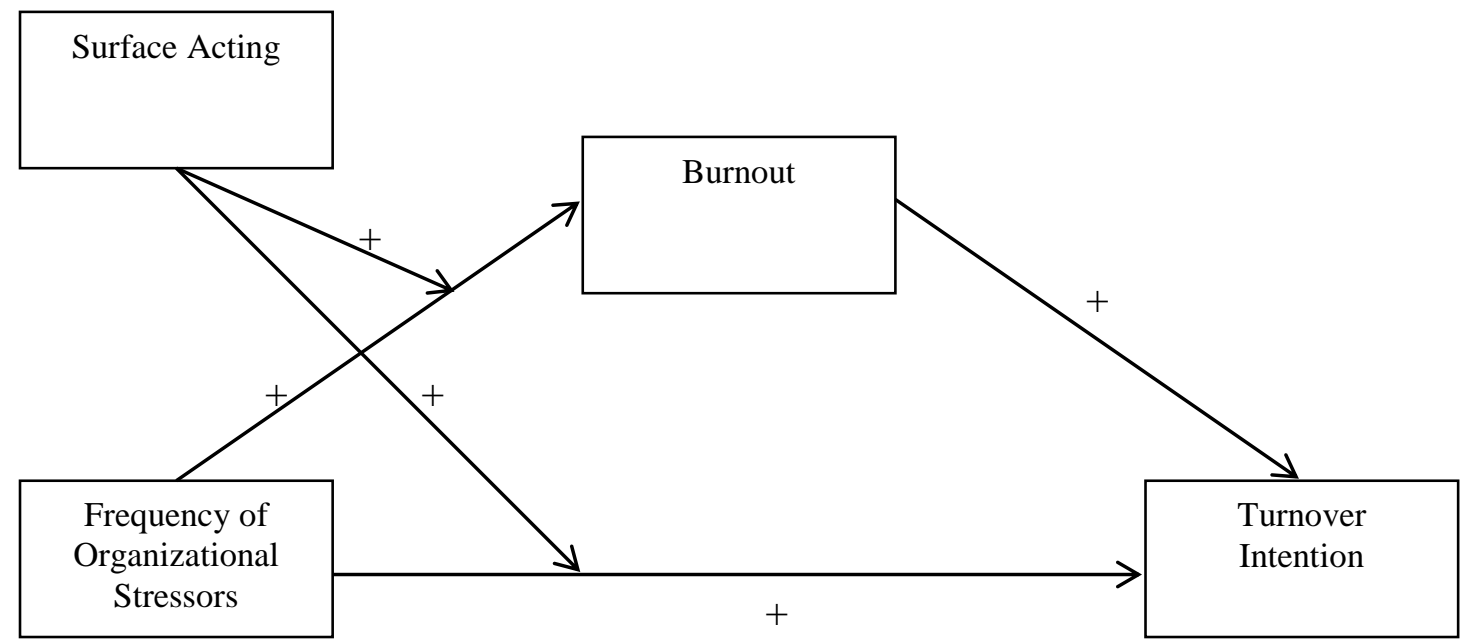

15 Figure 1. Hypothesised model for organizational stressors, surface acting, burnout, and turnover 16 intention. 
Table 1 Correlations and descriptive statistics

\begin{tabular}{|c|c|c|c|c|c|c|c|c|c|c|c|c|c|c|c|c|c|c|}
\hline Variables & 1 & 2 & 3 & 4 & 5 & 6 & 7 & 8 & 9 & 10 & 11 & 12 & 13 & 14 & 15 & 16 & 17 & 18 \\
\hline 1. OSISP Freq & - & & & & & & & & & & & & & & & & & \\
\hline 2. OSISP Int & $.86^{* *}$ & - & & & & & & & & & & & & & & & & \\
\hline 3. OSISP Dur & $.80 * *$ & $.91 * *$ & - & & & & & & & & & & & & & & & \\
\hline 4. OSISP G\&D & $.74 * *$ & $.68 * *$ & $.67 * *$ & - & & & & & & & & & & & & & & \\
\hline 5. OSISP L\&O & $.72 * *$ & $.63 * *$ & $.56^{* *}$ & $.59 * *$ & - & & & & & & & & & & & & & \\
\hline 6. OSISP $\mathrm{T} \& \mathrm{Cu}$ & $.79 * *$ & $.67 * *$ & $.63 * *$ & $.47 * *$ & $.51 * *$ & - & & & & & & & & & & & & \\
\hline 7. OSISP Co & $.74 * *$ & $.64 * *$ & $.62 * *$ & $.48 * *$ & $.46^{* *}$ & $.45^{* *}$ & - & & & & & & & & & & & \\
\hline 8. OSISP S & $.75^{* *}$ & $.62 * *$ & $.57 * *$ & $.39 * *$ & $.41 * *$ & $.59 * *$ & $.35 * *$ & - & & & & & & & & & & \\
\hline 9. Surface acting & $.26 * *$ & $.26 * *$ & $.25 * *$ & $.24 * *$ & $.23 * *$ & $.22 * *$ & $.19 * *$ & $.15^{* *}$ & - & & & & & & & & & \\
\hline 10. Deep acting & $.26 * *$ & $.27 * *$ & $.27 * *$ & $.26 * *$ & $.25 * *$ & $.23 * *$ & $.15^{* *}$ & $.18 * *$ & $.13 * *$ & - & & & & & & & & \\
\hline 11. Burnout & $.32 * *$ & $.29 * *$ & $.31 * *$ & $.28 * *$ & $.20 * *$ & $.28 * *$ & $.25^{* *}$ & $.19 * *$ & $.31 * *$ & .07 & - & & & & & & & \\
\hline 12. PEE & $.40 * *$ & $.40 * *$ & $.39 * *$ & $.39 * *$ & $.32 * *$ & $.30 * *$ & $.31 * *$ & $.22 * *$ & $.29 * *$ & $.20 * *$ & $.74 * *$ & - & & & & & & \\
\hline 13. SD & $.22 * *$ & $.17 * *$ & $.20 * *$ & $.13 * *$ & $.17 * *$ & $.21 * *$ & $.18 * *$ & $.15^{* *}$ & $.22 * *$ & .06 & $.84 * *$ & $.46^{* *}$ & - & & & & & \\
\hline 14. RA & $.14 * *$ & $.12 *$ & $.14 * *$ & $.13^{* *}$ & -.01 & $.17 * *$ & $.12 * *$ & .09 & $.21 * *$ & -.08 & $.74 * *$ & $.27 * *$ & $.51 * *$ & - & & & & \\
\hline 15. TI & $.19 * *$ & $.17 * *$ & $.18 * *$ & .09 & $.09 *$ & $.20 * *$ & $.13 * *$ & $.13 * *$ & .08 & .04 & $.37 * *$ & $.20 * *$ & $.38 * *$ & $.29 * *$ & - & & & \\
\hline 16. TI1 & $.18 * *$ & $.16^{* *}$ & $.16^{* *}$ & $.09 *$ & .07 & $.14 * *$ & $.16^{* *}$ & $.13 * *$ & .02 & -.03 & .37 & $.19 * *$ & $.40 * *$ & $.29 * *$ & $.74 * *$ & - & & \\
\hline 17. TI2 & $.12 *$ & $.10 *$ & $.12 * *$ & .02 & -.01 & $.15^{* *}$ & .07 & $.11^{*}$ & .03 & -.01 & $.26 * *$ & $.12 * *$ & $.28 * *$ & $.22 * *$ & $.88 * *$ & $.53 * *$ & - & \\
\hline 18. TI3 & $.21 * *$ & $.18 * *$ & $.19 * *$ & $.12 * *$ & $.17 * *$ & $.20 * *$ & $.15^{* *}$ & $.10 *$ & $.14 * *$ & $.11 *$ & $.37 * *$ & $.22 * *$ & $.37 * *$ & $.27 * *$ & $.82 * *$ & $.60 * *$ & $.52 * *$ & - \\
\hline$M$ & 1.54 & 1.60 & 1.48 & 1.75 & 1.19 & 1.74 & 1.36 & 1.67 & 2.56 & 2.81 & 11.39 & 10.97 & 10.46 & 12.75 & 7.70 & 1.88 & 3.19 & 2.63 \\
\hline SD & .82 & .88 & .92 & .98 & .83 & 1.01 & 1.21 & 1.33 & .90 & 1.04 & 3.05 & 4.01 & 4.02 & 3.57 & 4.73 & 1.56 & 2.19 & 1.93 \\
\hline
\end{tabular}

Note: G \& D: goals and development; L \& O: logistics and operations; T \& Cu: team and culture; Co: coaching; S: selection; PEE: physical and emotional exhaustion; SD: sport devaluation; RA: reduced accomplishment; TI: turnover intention. $* * p<.01 ; * p<.05$ (2-tailed) 
Table 2

Simple moderation results

\begin{tabular}{lcccc}
\hline Variable & $\begin{array}{c}b \\
\text { [LLCI, ULCI] }\end{array}$ & se & $t$ & $p$ \\
\hline Constant & 11.30 & .13 & 88.81 & .000 \\
Surface acting & {$[11.05,11.54]$} & .13 & 5.77 & .000 \\
OSISP Frequency & .88 & .15 & 5.67 & .000 \\
OSISP Freq x surface acting & {$[.58,1.18]$} & .17 & & .003 \\
\hline
\end{tabular}

Note. LLCI: lower limit confidence interval; ULCI: upper limit confidence interval.

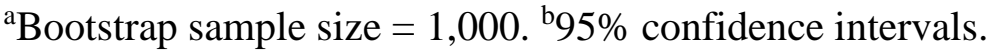




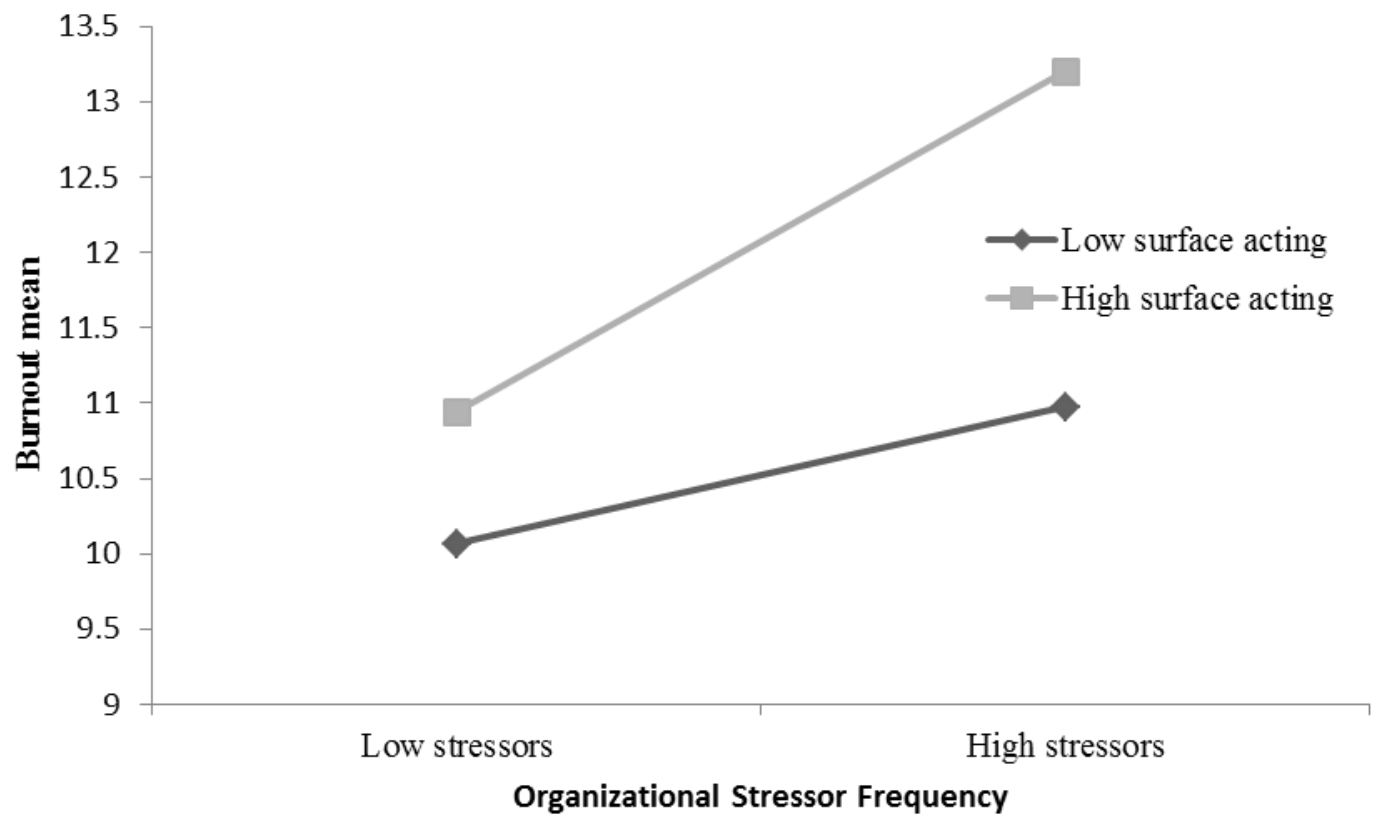

Figure 2. Plot of the interaction between the frequency of organizational stressors and surface acting in predicting burnout. 


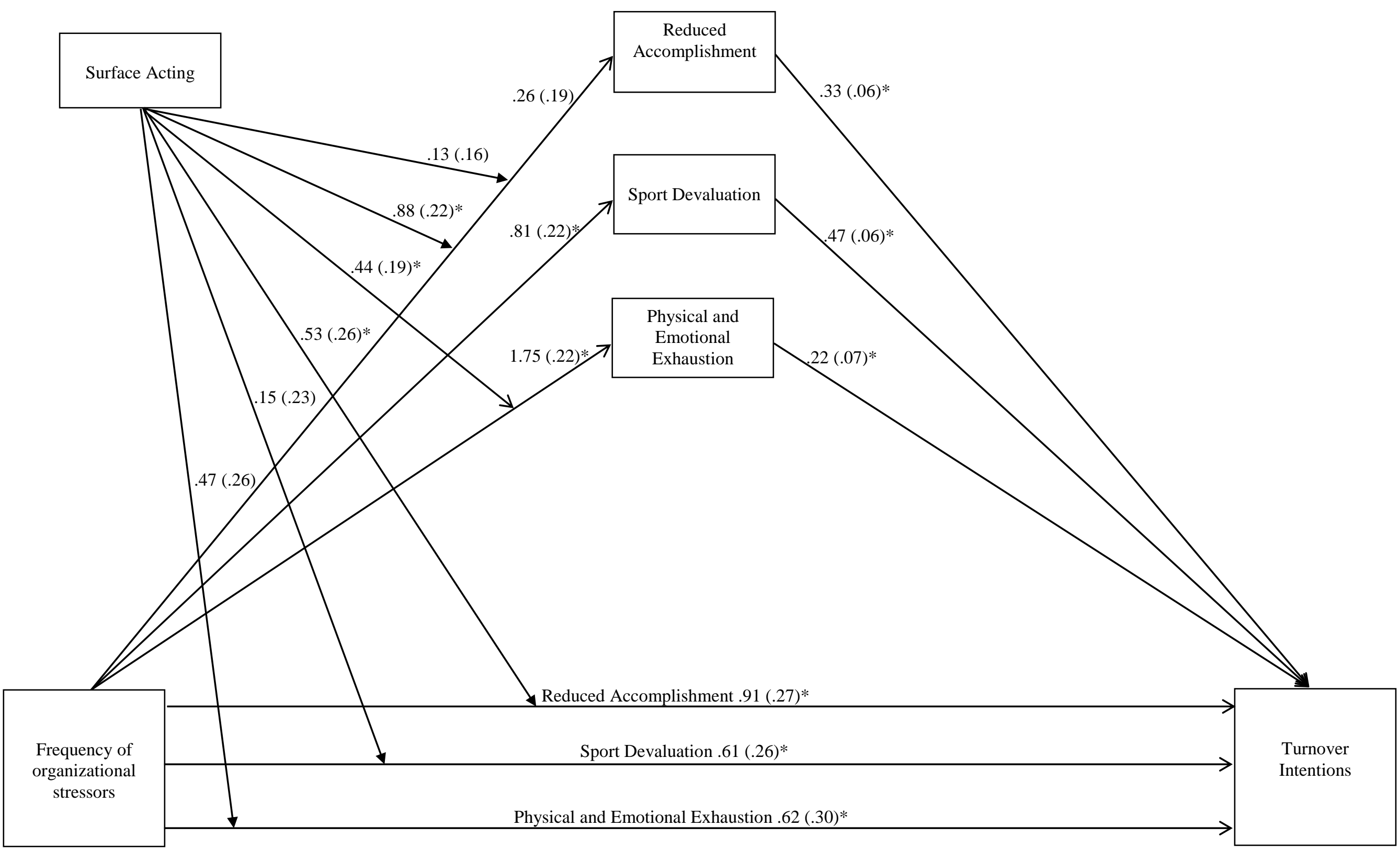

Figure 3. Moderated-mediation model with surface acting as a moderator. ${ }^{*} p<.05$ 
Table 3

Conditional indirect effects of organizational stressor frequency on turnover intentions (through burnout) at three levels of surface acting

$\begin{array}{llll}\text { Surface acting } & b(\mathrm{SE}) & \text { LL 95\% CI } & \text { UL 95\% CI }\end{array}$

\begin{tabular}{lccc}
\hline$-1 \mathrm{SD}(-.90)$ & $.33(.14)$ & .08 & .64 \\
$\mathrm{M}(.00)$ & $.57(.12)$ & .35 & .83 \\
$+1 \mathrm{SD}(.90)$ & $.81(.16)$ & .54 & 1.19
\end{tabular}

Note. LL: lower limit; CI: confidence interval; UL: upper limit; SD: standard deviation; M: mean.

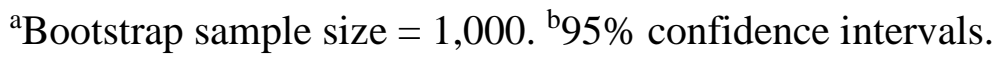


Table 4 Correlations and descriptive statistics

\begin{tabular}{|c|c|c|c|c|c|c|c|c|c|c|c|c|c|c|c|c|c|c|c|}
\hline Variables & 1 & 2 & 3 & 4 & 5 & 6 & 7 & 8 & 9 & 10 & 11 & 12 & 13 & 14 & 15 & 16 & 17 & 18 & 19 \\
\hline 1. OSISP Freq & - & & & & & & & & & & & & & & & & & & \\
\hline 2. OSISP Int & $.77 * *$ & - & & & & & & & & & & & & & & & & & \\
\hline 3. OSISP Dur & $.72 * *$ & $.90 * *$ & - & & & & & & & & & & & & & & & & \\
\hline 4. OSISP G\&D & $.69 * *$ & $.53 * *$ & $.53 * *$ & - & & & & & & & & & & & & & & & \\
\hline 5. OSISP L\&O & $.61 * *$ & $.48 * *$ & $.39 * *$ & $.47 * *$ & - & & & & & & & & & & & & & & \\
\hline 6. OSISP T\&Cu & $.78 * *$ & $.62 * *$ & $.60 * *$ & $.40 * *$ & $.41 * *$ & - & & & & & & & & & & & & & \\
\hline 7. OSISP Co & $.70 * *$ & $.52 * *$ & $.78 * *$ & $.33 * *$ & $.48 * *$ & $.41 * *$ & - & & & & & & & & & & & & \\
\hline 8. OSISP S & $.70 * *$ & $.56^{* *}$ & $.56 * *$ & $.48 * *$ & $.20 * *$ & $.52 * *$ & $.22 * *$ & - & & & & & & & & & & & \\
\hline $\begin{array}{l}\text { 9. Surface } \\
\text { Acting }\end{array}$ & $.24 *$ & $.22 *$ & $.22 *$ & $.40^{*}$ & .13 & .16 & .16 & .08 & - & & & & & & & & & & \\
\hline 10. Deep Acting & $.27 *$ & $.24 *$ & $.32 * *$ & .21 & .20 & $.22 *$ & $.21^{*}$ & .18 & $.30 * *$ & - & & & & & & & & & \\
\hline 11. TI (time1) & $.25^{*}$ & $.28 *$ & $.21 *$ & .04 & .14 & $.34 * *$ & .09 & .18 & .08 & .12 & - & & & & & & & & \\
\hline 12. TI1 & .17 & .20 & .08 & -.08 & .09 & $.23 *$ & .13 & .10 & -.04 & -.06 & $.70 * *$ & - & & & & & & & \\
\hline 13. TI2 & .13 & .19 & .11 & -.08 & -.03 & $.26^{*}$ & -.07 & .19 & -.07 & .06 & $.84 * *$ & $.49 * *$ & - & & & & & & \\
\hline 14. TI3 & $.30 * *$ & $.30 * *$ & $.27 * *$ & .10 & $.29 * *$ & $.36^{* *}$ & .21 & .13 & $.24^{*}$ & $.21^{*}$ & $.81 * *$ & $.54 * *$ & $.54 * *$ & - & & & & & \\
\hline 15. TI (time 2) & .01 & .11 & .05 & -.05 & .02 & .02 & -.04 & .04 & -.12 & .01 & $.49 * *$ & $.26^{*}$ & $.47 * *$ & $.36 * *$ & - & & & & \\
\hline 16. TI1 & .06 & .17 & .15 & -.04 & .01 & .08 & .02 & .09 & -.08 & -.01 & $.48 * *$ & $.27 * *$ & $.48 * *$ & $.30 * *$ & $.79 * *$ & - & & & \\
\hline 17. TI2 & -.06 & .03 & -.05 & -.07 & -.03 & -.09 & -.08 & -.02 & $-.25 *$ & -.08 & $.40 * *$ & $.25 *$ & $.41 * *$ & $.29 * *$ & $.79 * *$ & $.45^{* *}$ & - & & \\
\hline 18. TI3 & -.01 & .11 & .07 & -.10 & .03 & .03 & .01 & -.05 & -.04 & .09 & $.29 * *$ & .14 & $.29 * *$ & $.30 * *$ & $.83^{* *}$ & $.58 * *$ & $.55^{* *}$ & - & \\
\hline $\begin{array}{l}\text { 19. Actual } \\
\text { turnover }\end{array}$ & .08 & .10 & .09 & -.06 & .01 & .12 & .08 & -.12 & .08 & -.17 & $.27 *$ & $.27 *$ & $.22 *$ & $.21 *$ & $.46^{* *}$ & $.61 * *$ & $.24 *$ & $.24 *$ & - \\
\hline$M$ & 1.45 & 1.44 & 1.30 & 1.63 & 1.19 & 1.68 & 1.28 & 1.46 & 2.60 & 2.70 & 6.74 & 1.68 & 2.57 & 2.50 & 8.27 & 2.21 & 3.07 & 2.99 & .16 \\
\hline SD & .74 & .82 & .84 & .89 & .73 & .96 & 1.27 & 1.27 & .85 & 1.04 & 4.30 & 1.55 & 1.95 & 1.82 & 5.05 & 1.96 & 2.19 & 1.92 & .36 \\
\hline
\end{tabular}

Note: G \& D: goals and development; L \& O: logistics and operations; T \& Cu: team and culture; Co: coaching; S: selection; TI: turnover intention. $* * p<.01 ; * p<.05$ (2-tailed) 


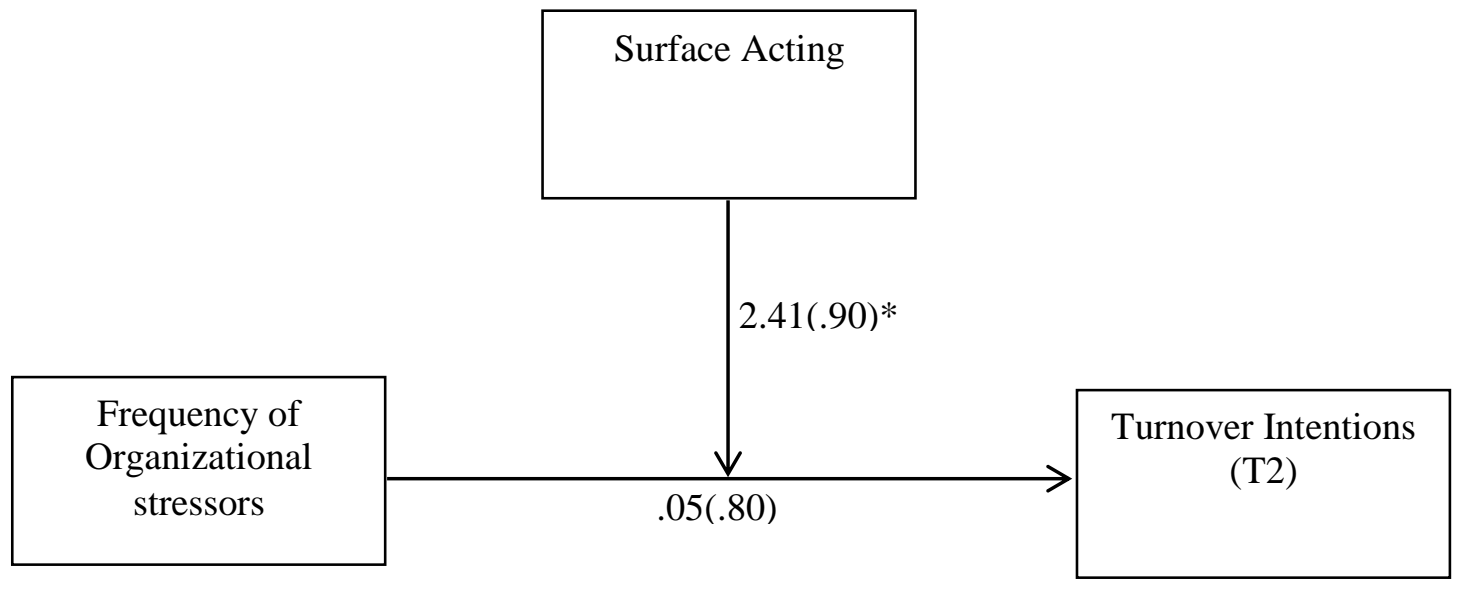

Figure 4. Moderation model for organizational stressor frequency, surface acting, and turnover intentions. ${ }^{*} p<.05$ 


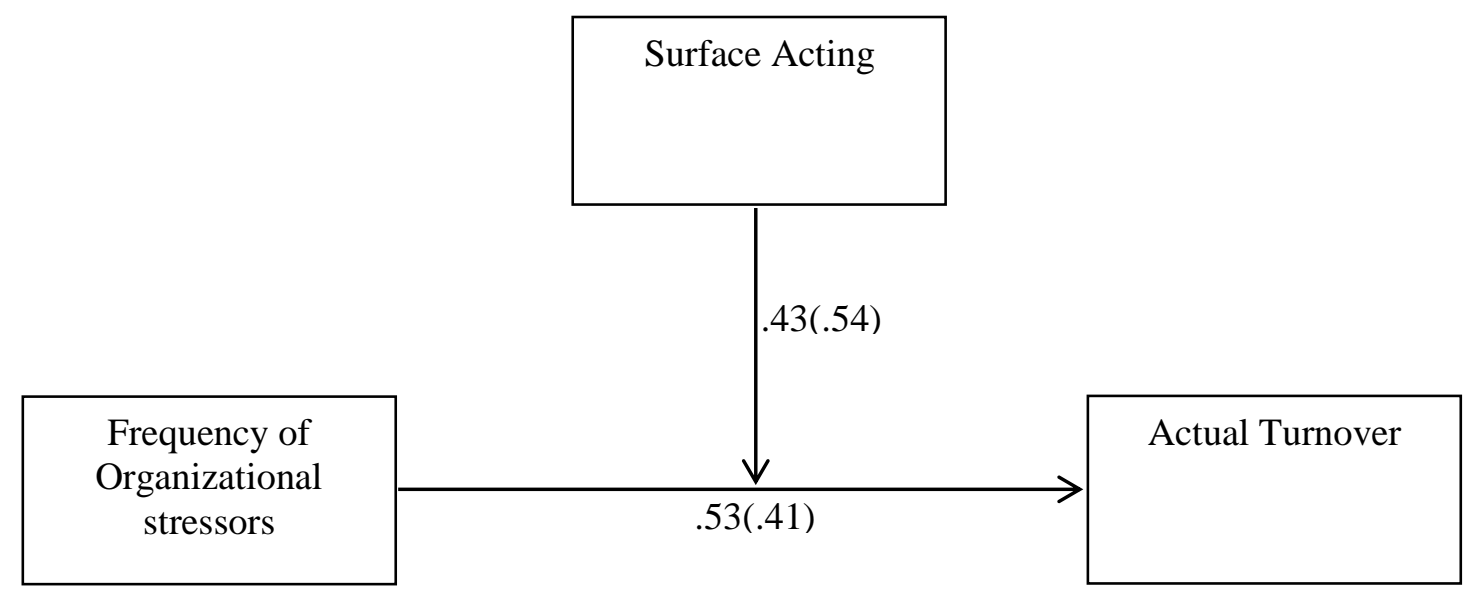

Figure 5. Moderation model for organizational stressor frequency, surface acting, and actual turnover. $* p<.05$ 
Table 5

Conditional indirect effects of the frequency of organizational stressors on turnover intentions (time 2) and actual turnover at three levels of surface acting

$\begin{array}{llll}\text { Surface acting } & b(\mathrm{SE}) & \text { LL 95\% CI } & \text { UL 95\% CI }\end{array}$

Turnover Intentions

$-1 \mathrm{SD}(-.85)$

$-2.01(1.31)$

$-4.60$

$\mathrm{M}(.00)$

$.05(.80)$

$-1.55$

1.64

$+1 \mathrm{SD}(.85)$

$2.11(.88)^{*}$

.36

3.85

Actual turnover

$-1 \mathrm{SD}(-.85)$

$.16(.56)$

$-.94$

1.26

M (.00)

$.53(.41)$

$-.28$

1.34

$+1 \mathrm{SD}(.85)$

$.90(.67)$

$-.41$

2.20

Note. LL: lower limit; CI: confidence interval; UL: upper limit; SD: standard deviation; M: mean.

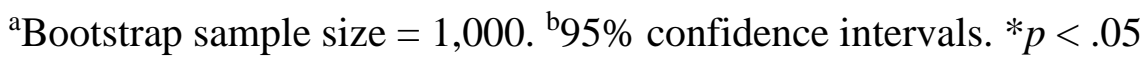

